PROCEEDINGS OF THE

AMERICAN MATHEMATICAL SOCIETY

Volume 27, Number 1, January 1971

\title{
LOCALLY FINITE SELF-INTERCHANGE GRAPHS
}

\author{
BENJAMIN L. SCHWARTZ AND LOWELL W. BEINEKE
}

Abstract. Graphs isomorphic to their interchanges are studied. Using prior results of more special cases, plus one new concept, it is possible to characterize all locally finite self-interchange graphs, finite and infinite, connected and disconnected, with loops and parallel edges admitted. All solutions are shown to be componentunions of graphs from six easily described classes.

1. Introduction. If $G$ is a (unoriented) linear graph, we define the interchange graph of $G, I(G)$ as the graph $H$ whose vertices are the edges of $G$; and two vertices in $H$ are consecutive iff the edges in $G$ are consecutive. (A loop is consecutive with itself [16].) A graph isomorphic with its interchange is called a self-interchange graph (SIG). In 1962, Ore [11] posed the problem of characterizing SIG's. Subsequently, progress has been made, under various combinations of hypotheses, e.g. graphs which were connected, finite, bounded degree, locally finite, loop-free, and/or having no parallel edges.

The relationships among the several cases has not always been clear. For example, there are connected SIG's of bounded degree that are not loop-free; but there are also finite-degree loop-free SIG's that are not connected [14], [13]. All locally finite loop-free SIG's are actually of bounded degree [6]; but it has previously been undetermined whether a locally finite SIG with loops must be of bounded degree. This question, and many other similar ones, are implicitly answered in the present paper, which solves the problem under the hypothesis only of local finiteness, a condition less restrictive than any previously solved case. The proof uses previous work, and one new idea ( $\$ 3)$.

2. Preliminaries. Let $\mathfrak{C}_{\boldsymbol{i}}$ denote an elementary chain of length $i$, $i=0,1,2,3, \cdots$. Let $\mathcal{L}$ be $\bigcup_{i=0}^{\infty} \mathfrak{C}_{i}$, where the $U$ denotes $C$-union of graphs. (The $C$-union, or component-union, of two graphs, $G$ and $H$, is the graph whose components are the components of $G$ and the components of $H$.) Let $\mathscr{D}_{i}$ be the graph comprising two loops joined by an elementary chain of length $i, i=0,1,2, \cdots$.

THeOREM 1. If $G$ is a loop-free locally finite SIG, then $G$ is the $C$ -

Received by the editors November 18, 1969.

AMS 1970 subject classifications. Primary 05C 99.

Key words and phrases. Graph theory, interchange graphs.

Copyright (C) 1971, American Mathematical Society 
union of graphs of the following forms:

(1) $\mathscr{L}$;

(2) a finite cycle of length $i, i=2,3,4, \ldots$;

(3) a one-way infinite elementary chain;

(4) a two-way infinite elementary chain.

This theorem is proved in [6].

Another prior result which we shall need is the following.

Lemma 1. If $G$ is an interchange graph, say $G=I(H)$; then $D_{0}$ is not a subgraph of $G$. Furthermore, for $i>0, D_{i}$ is a subgraph of $G$ iff $D_{i-1}$ is a subgraph of $H$.

These results are from [15], based on earlier work of Krausz [5] and Whitney [17].

THEOREM 2. If $G$ is $S I G$ with loops, then no component of $G$ has more than one loop.

Proof. Otherwise, there is a subgraph of the form $D_{i}$ in $G$. Let $i^{*}$ be the minimum value of $i$ for which $D_{i}$ is a subgraph of $G$. Then by Lemma 1 , we conclude that $i^{*}>0$. And by the same lemma, we have that $D_{i^{*}-1}$ is subgraph of $G$, contradicting the minimality of $i^{*}$.

\section{Loop-free transformation.}

Lemma 2. Let $G$ be a graph and $H$ its interchange graph. Assume that $G$ has a loop at vertex $v$ and that $x$ is the vertex in $H$ corresponding to that loop. Let $G_{n}^{\prime}$ be the graph obtained from $G$ by replacing the loop at $v$ by a new chain of length $n$, and let $H^{\prime}{ }_{n}$ be the graph obtained in the same way by replacing the loop at $x$. Then $H^{\prime}{ }_{n}$ is the interchange graph of $G^{\prime}{ }_{n+1}$ for all $n \geqq 0$.

Proof. The result follows easily by induction on $n$.

THEOREM 3. Let $G$ be a connected graph with exactly one loop. Let $v$ denote the vertex of the loop. Let $H$ be the interchange graph of $G$, with $x$ the vertex of $H$ corresponding to the loop. Let $G^{\prime}$ be the graph obtained from $G$ by replacing the loop at $v$ by $a$ new one-way infinite elementary chain, and let $H^{\prime}$ be the graph obtained in the same way by replacing the loop at $x$. Then $H^{\prime}$ is $I\left(G^{\prime}\right)$.

Proof. In Lemma 2, let $n$ increase without limit.

REMARK. If $\left\{G_{n}\right\}$ is a sequence of graphs satisfying $G_{n} \subset G_{n+1}$, then the concept $\lim _{n \rightarrow \infty} G_{n}$ is meaningful. In general, a vertex or edge is an element of $\lim G_{n}$ iff for some $N$, it is an element of all $G_{n}$ for which $n \geqq N$. A rigorous development of this idea occurs in [13], where the discussion shows the validity of the above limit-taking step. 
If $G$ is a graph with loops, define the Loop-Free Transformation (LFT) of $G$ as the graph $H$ which coincides with $G$, except that each loop is deleted (but not its vertex), and replaced by a one-way infinite elementary chain which attaches to $H$ at the vertex of the loop. In terms of this, a corollary to Theorem 3 can be stated in the following form:

CoRollary 1. For graphs in which no component has more than one loop, the operations of interchange and loop-free transformation commute.

Proof. For those components that have a loop, Theorem 3 gives the result. For those that have no loop, the LFT is the identity transformation.

THEOREM 4. If $G$ is a SIG with loops, and $H$ is the LFT of $G$, then $H$ is a loop-free $S I G$.

Proof. Since $G$ is a SIG, no component of $G$ has more than one loop, and we have $I(G)=G$. Take the LFT of both sides of this equation; then commute the LFT and I operations on the left-hand side. This gives $I(H)=H$.

\section{Main result.}

THEOREM 5. If $G$ is a locally finite SIG, then $G$ is the C-union of graphs of the following forms:

(1) $\mathfrak{L}$;

(2) finite cycles of length $i, i=2,3, \cdots$;

(3) a one-way infinite elementary chain;

(4) a two-way infinite elementary chain;

(5) a loop with a finite elementary chain (of any length $\geqq 0$ ) adjoined;

(6) a loop with a one-way infinite elementary chain adjoined.

Proof. By Theorem 4, the LFT of $G$ is a loop-free locally finite SIG, and hence, by Theorem 1, consists of graphs of the form (1), (2), (3), or (4). The only ones of these which may have had loops before being transformed by the LFT operations are (3) or (4). In case (3), a loop might have appeared in $G$ at any finite point on the chain. In case (4), a loop might have appeared in $G$ in place of a one-way chain; but not two loops, by Theorem 2. Hence graphs of the form of (5) and (6) are candidates. By direct calculation they are SIG's. This completes the proof.

This theorem brings order to the various special cases treated in the 
literature. It is now easy to characterize any subclass among the locally finite SIG's. For example, for loop-free SIG's, omit cases (5) and (6); for finite, connected SIG's, use only cases (2) and (5); for SIG's all of whose components are finite, include cases (1), (2), and (5); etc.

5. Remark. This proof obtains in an entirely new way the graph of form (5), the loop with finite chain adjoined. This graph is notable in being an early counterexample [3] to the conjecture that the only finite connected SIG's were cycles, and hence regular of degree 2. The existence of such a graph seems to have been largely neglected in the literature; although in [14] it was shown to be the unique nonregular finite connected SIG. Hence the new derivation is interesting in leading back to an old but little known result in finite connected graphs through the more impressive machinery of infinite and disconnected graphs.

\section{BIBLIOGRAPHY}

1. A. Andreatta, Sui singrammi finiti commutati di altri, Inst. Lombardo Accad. Sci. Lett. Rend. A 98 (1964), 133-156. MR 31 \#80.

2. G. Chartrand, The existence of complete cycles in repeated line-graphs. Bull. Amer. Math. Soc. 71 (1965), 668-670. MR 31 \#82.

3. Capt. Bernard Clark, USMC, Private communication, 1964.

4. Anna Maria Ghirlanda, Osservazioni sulle caratteristiche dei grafi o singrammi, Ann. Univ. Ferrara Sez. VII 11 (1962-65), 93-106. MR 32 \#7446.

5. J. Krausz, Démonstration nouvelle d'une thérème de Whitney sur les réseaux, Mat. Fiz. Lapok 50 (1943), 75-85. (Hungarian) MR 8, 284.

6. Livio Porcu, Sui grafi autocommutati, Inst. Lombardo Accad. Sci. Lett. Rend. A 100 (1966), 665-677. MR 34 \#7404.

7. V. Menon, The isomorphism betewen graphs and their adjoint graphs, Canad. Math. Bull. 8 (1965), 7-15. MR 30 \#5299.

8. - On repeated interchange graphs, Amer. Math Monthly 73 (1966), 986989. MR 34 \#2493.

9. - Repeated adjoints of graphs, Proc. Internat. Sympos. Theory of Graphs (Rome, 1966), Gordon and Breach, New York; Dunod, Paris, 1967, pp. 245-248. MR 36 \#6314.

10. L. Muracchini and A. Ghirlanda, Sul grafo commutato e sul grafo opposto di un grafo orientato, Atti. Sem. Mat. Fis. Univ. Modena 14 (1965), 87-97. MR 33 \#2570.

11. Oystein Ore, Theory of graphs, Amer. Math. Soc. Colloq. Publ., vol. 38, Amer. Math. Soc., Providence, R. I., 1962. MR 27 \#740.

12. A. van Rooij and $\mathrm{H}$. Wilf, The interchange graph of a finite graph, Acta. Math. Acad. Sci. Hungar. 16 (1965), 263-269. MR 33 \#3959.

13. Gert Sabidussi, Existence and structure of self-adjoint graphs, Math. Z. 104 (1968), 257-280. MR 37 \#2629.

14. B. L. Schwartz, On interchange graphs, Pacific J. Math. 27 (1968), 393-396. MR 38 \#3174. 
15. - Infinite self-interchange graphs, Pacific J. Math. 31 (1969), 497-504.

16. - Defining the interchange of a loop, Math. Mag. 43 (1970), 141-143.

17. H. Whitney, Congruent graphs and the connectivity of graphs, Amer. J. Math. 54 (1932), 150-168.

The Mitre Corporation, Westgate Research Park, Mclean, Virginia 22101

Purdue University, Fort Wayne, Indiana 46805 